\title{
Safety and Efficacy of Sacubitril/Valsartan Early Initiation in Hospitalized Patients with Heart Failure and Reduced Ejection Fraction
}

\author{
MOHAMMED MOANES M. MOHY EL DIN, M.D. and EL KAFOURY A. HAMDY, M.D.
}

The Department of Cardiology, Faculty of Medicine, Al-Azhar University, Egypt

\begin{abstract}
Background: Current guidelines approved Sacubitril/ valsartan as replacement therapy to angiotensin-convertingenzyme inhibitors or angiotensin-receptor-blockers for treatment of heart failure patients with reduced ejection fraction.

Aim of Study: The study was conducted to evaluate the safety, efficacy, and tolerability of sacubitril/valsartan (S/V) initiation in hospitalized patients.

Patients and Methods: This is a Prospective observational cohort study that included 191 patients who were hospitalized due to heart failure with reduced Ejection fraction (HFrEF) from March 2019 till June 2020. Sacubitril/valsartan was initiated during hospitalization and after hemodynamic stabilization. The study evaluated the following: Incidence of hypotensive events, the incidence of inpatient acute kidney injury and hyperkalemia, rate of discontinuation, and change in ejection fraction (EF), Brain natriuretic peptide (BNP), and $\mathrm{N}$-terminal Pro-B-type natriuretic peptide (NT-pro-BNP) from baseline and after one and three months from initiation.
\end{abstract}

Results: The mean age was $60.4 \pm 12.4$ years and $73.8 \%$ of patients were males. Of the studied group, $92.1 \%$ continued to take $\mathrm{S} / \mathrm{V}$, while $3.1 \%$ stopped medicine due to symptomatic hypotension, $2.6 \%$ due to financial issues, $1 \%$ due to AKI, and $1 \%$ due to hyperkalemia. After three months, only $5.8 \%$ of the patients tolerated the maximum dose (97-103) bid. For patients who continued to take $\mathrm{S} / \mathrm{V}$, there was a significant increase in EF at one- and three-month post-initiation, 28.4\% vs. $33.8 \%$ \& $28.4 \%$ vs. $39.4 \%$ respectively, $(p<0.001)$. On the other hand, there was a significant decrease in both BNP and NT-pro-BNP, $(p<0.001)$.

Conclusions: Early initiation of sacubitril/valsartan during hospitalization is safe and tolerable with good efficacy and minimal adverse effects.

Key Messages: Most hospitalized patients due to acute decompensated heart failure and reduced ejection fraction can tolerate initiation of sacubitril/valsartan after hemodynamic stabilization guided by strict blood pressure, creatinine, and potassium level monitoring.

No conflict of interest.

Correspondence to: Dr. Mohammed Moanes M. Mohy El Din E-Mail: MohammedMoanes.206@azhar.edu.eg
Key Words: Heart failure with reduced ejection fraction Angiotensin receptor - Neprilysin inhibitor Sacubitri l/valsartan.

\section{Introduction}

THE prognosis of Heart failure patients with reduced ejection fraction (HFrEF) is stillpoor despite therecent advances in the treatment modalities. It has been established that HFrEF isassociated with adverse short and long-term events despite the improvement of patient's symptoms achieved with standard medications [1]. Sacubitril/Valsartan (S/V) is a dual-acting Drug, the first component, Sacubitril is a pro-drug that, after activation, inhibits neprilysin, preventing natriuretic peptides degradation. The second component, valsartan, causes a simultaneous block of the renin-angiotensinaldosterone system (RAAS). This simultaneous action leads to an extended duration of the beneficial effects of natriuretic peptides and the prevention of RAAS's deleterious effects. These actions are associated with subsequent systemic vasodilation, a decrease of peripheral vascular resistance, increase in both diuresis and natriuresis with the resultant decrease in plasma volume [2]

S/V was approved by Food and Drug Administration (FDA) to be used in the treatment of chronic heart failure patients with reduced ejection fraction (HFrEF) with NYHA functional class II-IV. S/V is recommended to be used as replacement therapy to Angiotensin-converting enzymes inhibitors (ACEI) or angiotensin II receptor blockers (ARBs), in addition to the standard, anti-failure treatments including beta-blockers and mineralocorticoid receptors antagonists (MRA) [3]. Recently, S/V is useful in the treatment of apparently resistant hypertension in patients with heart failure and preserved ejection fraction (HFpEF), even in those 
patients with elevated blood pressure who are treated with at least four antihypertensive medications classes, including an MRA [4].

In 2016, S/V was recommended by the guidelines published by the European Society of Cardiology (ESC) as a class IB to be given for symptomatic patients despite the optimal guideline-directed medical therapy [5]. Also, the ACC/AHA/HFSA Focused Update Guidelines published in 2017 recommended that NYHA class II-III patients toleratingan (ACEI) or (ARBs) should be switched to $\mathrm{S} / \mathrm{V}$ as it reduces both the mortality and morbidity risks related to $\mathrm{HF}$ [6]. These recommendations were based on the PARADIGM-HF clinical trial, a randomized, double-blind one, compared the effect of S/V prescription versus enalapril in the symptomatic ambulatory stable patients with chronic HF. The trialconcluded that $\mathrm{S} / \mathrm{V}$ is better than Enalapril as S/V given group was associated with a lower incidence of both cardiovascular death and hospitalizations due to HF [7].

The possibility of prescribing S/V for in patients after stabilization of acute decompensation was addressed by two other trials whose primary results were released in 2018. The TRANSITION study, compared between prescribing S/V before discharge, and at least 24 hours or more after hemodynamic stabilization and prescribing it after discharge (within 1-14 days). The percentage of patients achieving a dose of $97 / 103 \mathrm{mg}$ twice a day at 10 weeks was the primary endpoint of this study. The secondary objective of the study was the percentage of patients who reached and maintained a doseof $100 \mathrm{and} /$ or 200mg twice a day, or any dose for at least 2 weeks and up to 10 weeks. Also, the study calculated the percentage of patients who permanently discontinued S/V during the same period. the study included 1002 subjects (500predischarge \& 502 post-discharge). TRANSITION results showed that nearly $50 \%$ of the patients with acute HF decompensation achieved the recommended $\mathrm{S} / \mathrm{V}$ target maximum dosewithin 10 weeks after stabilization. This concluded that $\mathrm{S} / \mathrm{V}$ initiation in hospitalized patients is well-tolerated and feasible [8]. In the second study, the PIONEER-HF study, hospitalized patients with HFrEF were randomized after hemodynamic stabilization either to receive $\mathrm{S} / \mathrm{V}$ with the maximum target dose (200mg twice daily) or enalapril with a dose of $10 \mathrm{mg}$ twice daily. The study included 881 patients, 440 of themreceived S/V, while 441 patients received enalapril. The study showed that S/V reduced NT-pro-BNP to a greater extent than enalapril. Moreover, this reduction was noted early afterone week from drug initiation. The study concluded that initiation of
SV in hospitalized patients was associated with a significantly greater decrease in the NT-pro-BNP level versus enalapril with no difference regarding the adverse drug events. Moreover, in-hospital S/V initiation was associated with fewer re-admission for HF at eight weeks when compared to enalapril [9].

The useful effects of S/V in HFrEF were confirmed in more than one recent real-life clinical study showing a significant decrease in cardiac death and HF rehospitalization with an improvement of left ventricular EF, systolic volume, and systolic pulmonary arterial pressure, renal function, and quality of life [10-13]. Moreover, a recent study compared the therapeutic effect of S/V and RAAS inhibitors alone and concluded that the therapeutic effects of S/V are correlated with LVEF with more benefits, for hospitalized patients, and even in patients with heart failure with mildly reduced ejection fraction. The therapeutic benefits extended to a higher LVEF value in women more than in men [14]

Our study was conducted to assess the daily clinical practice safety and efficacy of early initiation of Sacubitril/Valsartanin hospitalized patients with HFrEF. This assessment was based on clinical, laboratory, echocardiographic parameters as well as on clinical short-term outcomes.

\section{Patients and Methods}

The study included 191 out of 201 hospitalized patients with reduced EF due to decompensated heart failurefrom March 2019 to June 2020. Ten patients were excluded from the study, 4 patients died during hospitalization and 6 patients were lost during follow-up with missing data. All patients received sacubitril/valsartan in an adjustable dose according to baseline blood pressure during hospitalization, at least 24 hours from hemodynamic stabilization. The study evaluated the following: the incidence of hypotensive events during hospitalization, acute kidney injury and hyperkalemia, the rate of discontinuation and its cause, and change in ejection fraction (EF), Brain Natriuretic Peptide (BNP), and N-terminal Pro-B-type natriuretic peptide (NT-pro-BNP) during follow-up after one month and three months from initiation.

\section{Statistical analysis used:}

SPSS version 22 software (IBM, Inc., Armonk, NY) was used for statistical analysis of the collected data. Numerical variables were expressed as mean and SD. Chi-square tests were used for the analysis of the relation between two nominal variables. 
Paired-Samples " $t$ "-test was used to evaluate the change of EF, BNP, and N-T pro-BNB. A $p$-value less than 0.05 is considered statistically significant.

\section{Results}

The data included was reviewed, categorized, and analyzed. The Baseline characteristics of the studied population are shown in (Table 1).

Table (1): Baseline demographics and clinical characteristics.

\begin{tabular}{ll}
\hline Baseline variable & $\mathrm{N}=191$ \\
\hline Age (years), mean $\pm \mathrm{SD}$ & $60.3 \pm 12.3$ \\
Male sex, n (\%) & $141(73.8)$ \\
Hypertension, n (\%) & $89(46.6)$ \\
AF, $\mathrm{n}(\%)$ & $29(15.2)$ \\
Stroke, $\mathrm{n}(\%)$ & $8(4.2)$ \\
Type of Heart failure: & \\
$\quad$ Ischemic HF, $\mathrm{n}(\%)$ & $86(45)$ \\
$\quad$ Dilated Cardiomyopathy, $\mathrm{n}(\%)$ & $98(51.3)$ \\
$\quad$ Peripartum Cardiomyopathy, $\mathrm{n}(\%)$ & $7(3.7)$ \\
NYHA Class, $n$ (\%): & \\
$\quad$ III & $104(54.5)$ \\
$\quad$ IV & $87(45.5)$ \\
(CRTD), $\mathrm{n}(\%)$ & $12(6.3)$ \\
Index heart failure admission, $\mathrm{n}(\%)$ & $63(33)$ \\
Hospital day initiated, mean $\pm \mathrm{SD}$ & $3.6 \pm 1.3$ \\
Length of stay (days), mean $\pm \mathrm{SD}$ & $6.4 \pm 1.7$ \\
S Cr (day of initiation), mean & $1.09 \mathrm{mg} / \mathrm{dL}$ \\
S Cr (Peak), mean & $1.19 \mathrm{mg} / \mathrm{dl}$ \\
Serum potassium (Baseline), mean & $3.8 \mathrm{mEq} / \mathrm{L}$ \\
Serum potassium (Peak), mean & $4.1 \mathrm{mEq} / \mathrm{L}$ \\
BNP (admission), mean & $1163 \mathrm{pg} / \mathrm{nL}$ \\
BNP (discharge), mean & $559 \mathrm{pg} / \mathrm{nL}$ \\
NT-Pro BNP (admission), mean & $5360 \mathrm{pg} / \mathrm{Nl}$ \\
NT-Pro BNP (discharge), mean & $1877 \mathrm{pg} / \mathrm{nL}$ \\
\hline -At baseline &
\end{tabular}

- At baseline, the mean age was $60.4 \pm 12.4$ years, and 141 (73.8\%) of the studied patients were males.

The baseline means left ventricular EF (LVEF) was 28.4. Regarding NYHA class, 87 (45.5\%) of the included patients were in NYHA functional class IV on admission, while $104(54.5 \%)$ of the patients were in NYHA functional class III. There were $63(33 \%)$ patients with index hospitalization, while $128(67 \%)$ patients had a history of the previous hospitalization. All included patients were not prescribed $\mathrm{S} / \mathrm{V}$ before admission. Heart failure was ischemic in 86 patients (45\%) of the total studied patients. The mean Body Mass Index was $31.9 \pm 5.9,89(46.6 \%)$ patients were hypertensive, and $45(23.6 \%)$ patients were diabetic. Atrial fibrillation was the rhythm in $29(15.2 \%)$ of the included patients, while $4.2 \%$ of the studied population had a history of Stroke. The average hospitalstay length was $6.4 \pm 1.7$ days, while the average time of initiation of $\mathrm{S} / \mathrm{V}$ during hospitalization in days was 3.6 \pm 1.3 . Twelve patients representing $6.3 \%$ of thestudied population had cardiac resynchronization therapy defibrillator (CRT-D). Regarding baseline medications, as shown in (Table 2) there were $(67 \% \mathrm{~N}=129)$ patients on either (ACEI) or (ARBs), and in all these patients, S/V was not initiated except after at least 36 hours from stopping of ACEI or ARBs.

Table (2): Baseline medications.

\begin{tabular}{lc}
\hline Drug name & No./Percentage \\
\hline Beta-Blocker & $171(89.5)$ \\
ACEI or ARBs & $129(67 \%)$ \\
Mineralocorticoid antagonist & $143(74.9 \%)$ \\
\hline
\end{tabular}

Angiotensin-Converting Enzyme Inhibitor (ACEI).

Angiotensin Receptor Blockers (ARBs).

Table (3) shows the initial dose and frequency of Sacubitril/valsartan. Nine patients $(4.7 \%)$ were initiated on a dose of 24-26mg once daily which is lower than the standard starting dose of $24-26 \mathrm{mg}$ twice daily, due to baseline borderline blood pressure. Forty-four patients (23\%) were started on $49-51 \mathrm{mg}$ twice daily owing to their high blood pressure on presentation. The remaining patients (138) representing $(72.3 \%)$ were started on the standard dose of 24-26mg twice daily.

Table (3): Sacubitril/valsartan starting dose and frequency.

\begin{tabular}{cl}
\hline Starting Dose and Frequency, $\mathrm{n} \mathrm{( \% )}$ & $\mathrm{N}=191$ \\
\hline 24-26 mg once daily & $9(4.7)$ \\
24-26 mg twice daily & $138(72.3)$ \\
$49-51 \mathrm{mg}$ twice daily & $44(23.0)$ \\
\hline
\end{tabular}

Table (4) summarizes the mean daily dose of furosemide in $\mathrm{mg}$ per day calculated on admission, discharge, and during follow-up.

Table (4): Mean diuretic dose.

\begin{tabular}{cc}
\hline Mean diuretic Dose & $($ Mean \pm SD) \\
\hline At baseline & $66.7 \pm 18.1$ \\
At discharge & $69.5 \pm 29.8$ \\
At 1 month & $47.2 \pm 13.9$ \\
At 3 months & $30.3 \pm 15.8$ \\
\hline
\end{tabular}

Fig. (2) shows that the total number of patients who continued to take S/V was $176(92.1 \%)$ ). After three months of follow-up, $160(83.8 \%)$ patients were on $49-51 \mathrm{mg}$ twice daily, while five patients (2.6\%) failed to increase the dose and remained on $24-26 \mathrm{mg}$ bid. On the other hand, only $11(5.8 \%)$ patients tolerated the maximum dose (97-103) bid, (Fig. 1). 


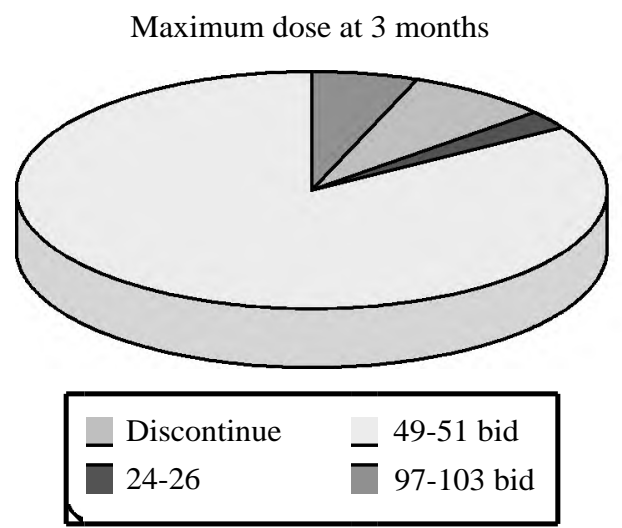

Fig. (1): Maximum dose of S/V after 3 months.

Of the total studied population, 15 patients (7.9\%) stopped S/V during hospitalization, 6 patients $(3.1 \%)$ due to symptomatic hypotension, five patients $(2.6 \%)$ due to financial issue, two patients (1\%) due to AKI, while the remaining two patients (1\%) stopped S/V due to hyperkalemia (Fig. 2).

Table (5) shows the relation ship between age, body mass index, and history of hypertension on

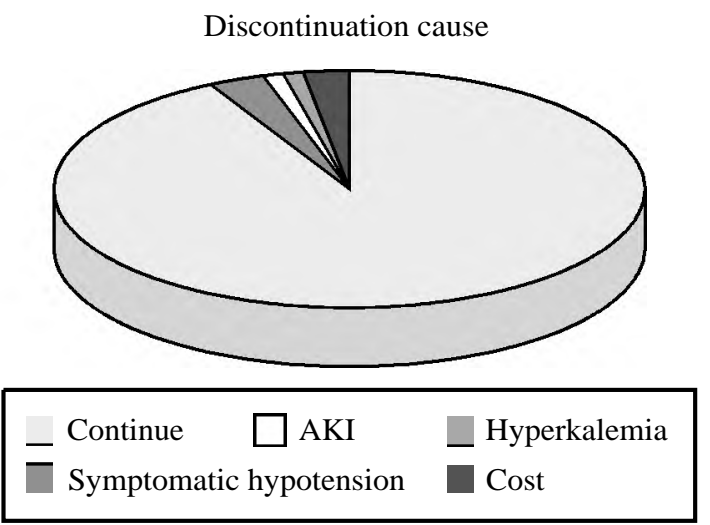

Fig. (2): Discontinuation Rate and cause per total number of population (AKI, acute kidney injury).

one side and the occurrence of adverse drug effects on the other side.

It was noted that absent history of hypertension was a highly significant variable in patients who developed hypotension with a highly significant $p$-value. Hyperkalemia happened in older patients with a significant $p$ value (0.02).

Table (5): Relation between adverse drug effects of S/V and baseline characteristics.

\begin{tabular}{|c|c|c|c|c|c|c|c|c|c|}
\hline \multirow{2}{*}{ Variable } & \multicolumn{3}{|c|}{ Hypotension } & \multicolumn{3}{|c|}{ Hyperkalemia } & \multicolumn{3}{|c|}{ AKI } \\
\hline & Yes (56) & No (135) & $\boldsymbol{p}$-value & Yes (2) & No (189) & $p$-value & Yes (2) & No (189) & $\boldsymbol{p}$-value \\
\hline Age mean & $57.8 \pm 12.5$ & $61.4 \pm 12.1$ & 0.06 & $71.5 \pm 2$ & $60 \pm 12.3$ & 0.02 & $68 \pm 18.3$ & $60.2 \pm 12.3$ & 0.381 \\
\hline BMI mean & $31.6 \pm 6$ & $32 \pm 5.8$ & 0.69 & $29.30 \pm 9.4$ & $31.9 \pm 5.9$ & 0.529 & $38.2 \pm 3.9$ & $31.8 \pm 5.9$ & 0.134 \\
\hline Hypertension history & $6(0.11 \%)$ & $83(61 \%)$ & 0.0001 & 2 & 187 & 0.216 & 0 & 189 & 0.5 \\
\hline
\end{tabular}

There was a statistically significant increasein $\mathrm{EF}$ at both onemonth, and three months postinitiation, $28.4 \%$ vs. $33.8 \%$ \& $28.4 \%$ vs; $39.4 \%$ respectively, with a highly significant $p$-value $(p<0.001)$. On the other hand, a significant reduction in both BNP and NT-pro-BNP was observed at both 1 and three months of follow-up $(p<0.001)$, (Table 6).

Table (6): Secondary efficacy.

\begin{tabular}{llllll}
\hline Outcome & Admission & \multicolumn{1}{c}{1 month } & $p$-value & \multicolumn{1}{c}{3 month } & $p$-value \\
\hline EF Mean & $28.49 \pm 4.78$ & $33.86 \pm 4.13$ & $<0.0001$ & $39.36 \pm 4.28$ & $<0.0001$ \\
BNB & $1161 \pm 172.3$ & $557 \pm 130$ & $<0.0001$ & $308 \pm 59$ & $<0.0001$ \\
Pro-BNB & $5411 \pm 1798$ & $1899 \pm 650$ & $<0.0001$ & $426 \pm 74$ & $<0.0001$ \\
\hline
\end{tabular}

\section{Discussion}

Our study was done to review and evaluate the real-world experience of starting S/V early during hospitalization in patients with HFrEF. PIONEERHF and TRANSITION trials were the two trials evaluating the initiation of $\mathrm{S} / \mathrm{V}$ in hospitalized patients. The PIONEER-HF trial was comparing two different drugs (sacubitril/valsartan versus enalapril) in hospitalized patients, while the TRANSITION trial was comparing between predischarge initiation of S/V and post-discharge initiation of the same medicine. Our study included a smaller number of patients (191) than the number included in PIONEER-HF (881) and transition (99 1) trials. On admission, $45.5 \%$ of patients were in NYHA class IV, and this is a much higher percentage than the percentage included in the PIONEER-HF trial 
(8.9\%) and TRANSITION trial (1.1\%). This means that our study included more symptomatic patients. This can be interpreted by the fact that these trial's inclusion criteria were stricter including more stable patients. In our study, index HF admission was $33 \%$ which is slightly lower than the percentage of index heart failure admission in the PIONEERHF trial (34.6\%) and much lower than thatpercentage in the TRANSITION trial $(51 \%)$. Our studied patients were younger (mean age 60) versus 66.8 and 62 in the TRANSITION and the PIONEERHF studies respectively. The percentage of male patients in our study is $73.8 \%$ which is comparable to the percentage in both PIONEER-HF (72\%) and TRANSITION $(75.1 \%)$ trials. The percentage of previously treated patients with ARBs or an ACEI in our study was (67\%) (Higher than) the percentage in PIONEER-HF $(47.9 \%)$. There was $24.3 \%$ of included patients in the TRANSITION trial were ARBs/ACEI naïve.

Our baseline means LVEF was $28.4 \%$, which is comparable to the mean EF at baseline in the TRANSITION trial (29\%). The median EF in the PIONEER-HF trial in the S/V group was $25 \%$. Despite the percentage of our hypertensive patients (46.6\%) was lower than that in the TRANSITION trial $(75.4 \%)$, the mean SBP at baseline in our study was $144 \mathrm{mmHg}$, which is higher than the baseline mean SBP in the TRANSITION trial $(124 \mathrm{mmHg})$. This can be explained by the fact that hypertension was more severe in our studied hypertensive group than the one included in the TRANSITION trial. Our mean length of stay was 6.4 days which is longer than the length of stay in the PIONEER-HF trial (5.2 days).

The total number of patients, in our study, who continued to take S/V after 3 months of follow-up was $176(92.1 \%)$, while 15 patients $(7.9 \%)$ of the total studied group stopped S/V during hospitalization. This rate is lower than the discontinuation rate in the TRANSITION trial (12.9\%). In the PIONEER-HF trial; $20 \%$ of the patients in each treatment arm had discontinued treatment by week 8 , mostly due to adverse events. In our study, it was noted that non-hypertension was a highly significant variable in patients who developed hypotension with a highly significant $p$-value and hyperkalemia happened in older patients with a significant $p$-value $(p=0.02)$. Seven patients only $(0.7 \%)$ in the TRANSITION study discontinued treatment permanently due to hypotension, with six patients from the pre-discharge group. This indicates the importance ofhemodynamic stabilization before initiating treatment with sacubitril/valsartan. Also, slow up-titration to target dose is recommended in the highly vulnerable postAcute decompensated HF phase. The discontinuation rate was higher in the PIONEER-HF however,a slightly higher proportion of patients in the PIONEER-HF tolerated the maximum target dose.

In our study, significant improvement in EF was observed at both one month and three months; post-initiation, $(28.4 \%$ vs. $33.8 \%) \&(28.4 \%$ vs. $39.4 \%$ ) respectively, with a highly significant $p$ value $(p<0.001)$. On the other hand, a significant reduction in BNP and NT-pro-BNP at both one and three months of follow-up $(p<0.001)$ was observed.

In our study, only $5.8 \%$ of patients tolerated the target dose of $\mathrm{S} / \mathrm{V}$, while about $48 \%$ of patients achieved the target dose in the TRANSITION trial, which is slightly lower than that proportion achieved in the PIONEER-HF (55\%). The possible reason for this difference is the smaller number enrolled in our study $[\mathbf{7 , 8 ]}$.

There is a Multicentre registry, published in ESC Heart Failure Journal in 2019, that concluded that $\mathrm{S} / \mathrm{V}$ treatment can be safely started during hospitalization in daily clinical practice with no evidence of increased risk of hypotension, worsening of renal function, and hyperkalemia. This multicentre registry included 527 patients and compared between in-hospital initiation of $S / V, 100$ patients (19\%) and outpatient initiation, 427 outpatients $(81 \%)$. According to this registry, the initiation of $\mathrm{S} / \mathrm{V}$ in outpatients is an independent predictor for the achievement of a higher dose. All-cause admissions during follow-up were more frequent in inpatients but with no relevant differences in all-cause mortality. There was no significant difference in the discontinuation rate, 17 inpatients ( $17 \%$ ) vs. 49 outpatients ( $11.5 \%), p=0.13$. There was no difference regarding the incidence of drug adverse events: Hypotension, 16 inpatients (16\%) vs. 71 outpatients $(16.7 \%), p=0.88)$, deteriorating renal function, 7 inpatients $(7.0 \%)$ vs. 29 outpatients $(6.8 \%), p=0.94)$, and hyperkalaemia, 1 inpatient (1.0\%) vs. 21 outpatients $(4.9 \%)$, $p=0.09$. All these data confirm the safety and tolerability of initiation of S/V during hospitalization after hemodynamic stabilization [15]

\section{Limitations of the study:}

Our study has many limitations: First, it is a small sample size. Second, no control group enrolled to exclude other possible factors that may have been affected the results. Third, it is difficult to attribute the improvement in ejection fraction to sacubitril/valsartan alone, since other HF therapies may contribute to the improvement. 
Despite these limitations, our study results are useful in providing additional data based on the routine daily clinical practice about the safety, efficacy, and tolerability of sacubitril/valsartan. In summary, there are Important parameters to be considered when initiating S/V therapy including frequent blood pressure monitoring and daily assessment of serum creatinine and serum potassium. Based on our study results, Sacubitril/valsartan can be initiated safely in admitted patients with acute decompensated heart failure and low EF after hemodynamic stabilization.

\section{References}

1- HANI N. SABBAH: Silent disease progression in clinically stable heart failure. Eur. J. Heart Fail, 4 (19): 469-78, 2017.

2- KOBALAVA Z., AVERKOV O., MERAY I., et al.: Natriuretic peptide inhibition in the presence of angiotensin receptor blockade following short-term treatment with LCZ696 in heart failure patients: Effect on ANP, BNP, NT-proBNP, and cGMP. Eur. Heart J., 32: 784-785, 2011.

3- SAUER A.J., COLE R., JENSEN B.C., et al.: Practical guidance on the use of sacubitril/valsartan for heart failure. Heart Fail Rev., 24 (2): 167-176, 2019.

4- ALICE M. JACKSON, PARDEEP S. JHUND, INDER S. ANAND, et al.: Sacubitril/valsartan as a treatment for apparent resistant hypertension in patients with heart failure and preserved ejection fraction. European Heart Journal, 00: 1-13, 2021.

5- PONIKOWSKI P., VOORS A.A., ANKER S.D., et al.: 2016 European Society of Cardiology (ESC) Guidelines for the diagnosis and treatment of acute and chronic heart failure: The Task Force for the diagnosis and treatment of acute and chronic heart failure of the European Society of Cardiology (ESC) Developed with the special contribution of the Heart Failure Association (HFA) of the ESC. Eur Heart J., 37 (27): 2129-200, 2016.

6- YANCY C.W., JESSUP M., BOZKURT B., et al.: 2017 ACC/AHA/HFSA Focused Update of the 2013 AC$\mathrm{CF} / \mathrm{AHA}$ guideline for the management of heart failure:
A report of the American College of Cardiology/American Heart Association Task Force on Clinical Practice Guidelines and the Heart Failure Society of America. J. Am. Coll. Cardiol., 70: 776-803, 2017.

7- McMURRAY J.J., PACKER M., DESAI A.S., et al.: Angiotensin-neprilysin inhibition versus enalapril in heart failure. N. Engl. J. Med., 371 (11): 993-1004, 2016.

8- WACHTER R., SENNI M., BELOHLAVEK J., et al.: Initiation of sacubitril/valsartan in hemodynamically stabilized heart failure patients in hospital or early after discharge: Primary results of the randomized TRANSITION study. European Heart Journal, Aug. 21 (8): 998$1007,2019$.

9- VELAZQUEZ E.J., MORROW D.A., DeVORE A.D., et al.: Angiotensin-neprilysin Inhibition in acute decompensated heart failure. N. Engl. J. Med., 380: 539-48, 2019.

10- SPANNELLA F., MARINI M., GIULIETTI F., et al.: Renal effects of sacubitril/valsartan in heart failure with reduced ejection fraction: A real-life 1-year follow-up study. Intern Emerg. Med., 14: 1287-97, 2019.

11- MENTZ R.J., XU H., O'BRIEN E.C., et al.: PROVIDEHF primary results: Patient-reported outcomes investigation following initiation of drug therapy with entresto (sacubitril/valsartan) in heart failure. Am. Heart J., 230: 35-43, 2020.

12- POLITO M.V., SILVERIO A., RISPOLI A., et al.: Clinical and echocardiographic benefit of sacubitril/valsartan in a real-world population with $\mathrm{HF}$ with reduced ejection fraction. Sci. Rep., 10: 6665, 2020.

13-ROBERT J. MENTZ, HAOLIN XU EMILY C. O'BRIEN, et al.: PROVIDE-HF primary results: Patient-Reported Outcomes investigation following Initiation of Drug therapy with Entresto (sacubitril/valsartan) in heart failure. American Heart Journal, December, 230: 35-43, 2020.

14- SCOTT D. SOLOMON, MUTHIAH VADUGANATHAN, BRIAN L. CLAGGETT, et al.: Sacubitril/Valsartan Across the Spectrum of Ejection Fraction in Heart Failure. Circulation, 141: 352-361, 2020.

15- LÓPEZ-AZOR J.C., VICENT L., VALERO-MASA M.J., et al.: Safety of sacubitril/valsartan initiated during hospitalization: Data from a non-selected cohort. ESC Heart Fail., 6: 1161-6, 2019. 


\section{سلامة وفعالية البدء المبكر لساكيوبيتريل / فالسارتان للمرضى المنومين

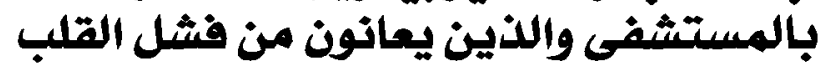

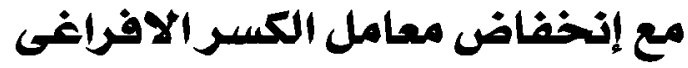

وافقت ادارة الأغذية والعقاقير الأمريكية مؤخراً على استعمال عقار الساكيويتريل / فالسارتان كعلاج بديل لمثطات الإنزيم المحل

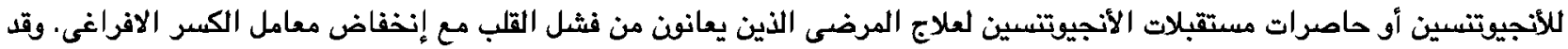

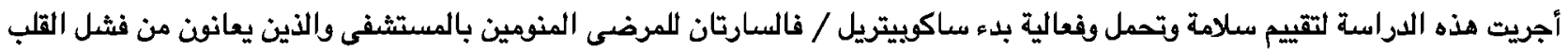

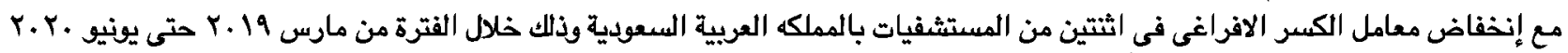

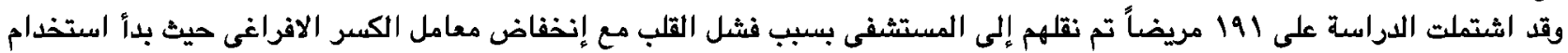

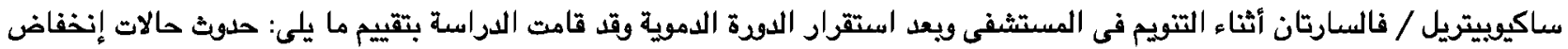

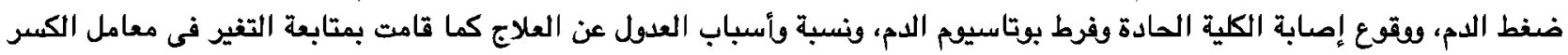

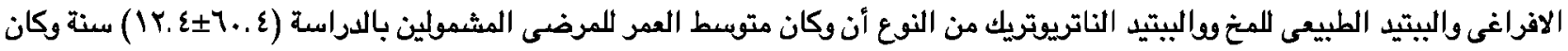

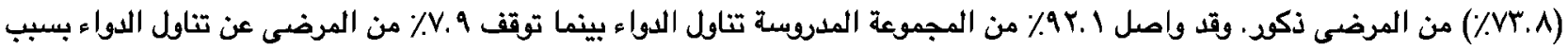

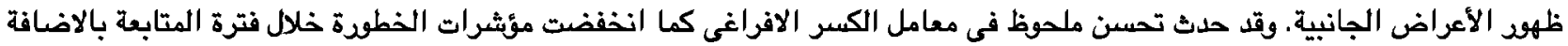
إلى تحسن ملحوظ في الأعراض.

وقد خلصت الدراسة إلى أن البدء المبكر فى إستعمال ساكيوبيتريل / فالسارتان يعد آمناً مع فعالية جيدة وآثار جانبية ثليلة. 